\title{
Vonoprazan for treatment of gastroesophageal reflux: pharmacodynamic and pharmacokinetic considerations
}

\author{
Edoardo Savarino, Irene Martinucci, Manuele Furnari, Gaia Pellegatta, \\ Alessandro Moscatelli, Giorgia Bodini, Elisa Marabotto, Vincenzo Savarino, \\ Nicola de Bortoli \& Corrado Blandizzi
}

To cite this article: Edoardo Savarino, Irene Martinucci, Manuele Furnari, Gaia Pellegatta, Alessandro Moscatelli, Giorgia Bodini, Elisa Marabotto, Vincenzo Savarino, Nicola de Bortoli \& Corrado Blandizzi (2016): Vonoprazan for treatment of gastroesophageal reflux: pharmacodynamic and pharmacokinetic considerations, Expert Opinion on Drug Metabolism \& Toxicology, DOI: 10.1080/17425255.2016.1214714

To link to this article: http://dx.doi.org/10.1080/17425255.2016.1214714

Accepted author version posted online: 18 Jul 2016.

Published online: $18 \mathrm{Jul} 2016$.

Submit your article to this journal $\sqsubset$

Q View related articles $\sqsubset$

View Crossmark data $[\pi$ 
Publisher: Taylor \& Francis

Journal: Expert Opinion on Drug Metabolism \& Toxicology

DOI: $10.1080 / 17425255.2016 .1214714$

\section{REVIEW}

Vonoprazan for treatment of gastroesophageal reflux: pharmacodynamic and pharmacokinetic considerations

Edoardo Savarino $^{1}$, Irene Martinucci ${ }^{2}$, Manuele Furnari ${ }^{3}$, Gaia Pellegatta ${ }^{3}$, Alessandro Moscatelli ${ }^{3}$, Giorgia Bodini $^{3}$, Elisa Marabotto ${ }^{3}$, Vincenzo Savarino ${ }^{3}$, Nicola de Bortoli ${ }^{2}$, Corrado Blandizzi $^{4}$

${ }^{1}$ Division of Gastroenterology, Department of Surgery, Oncology and Gastroenterology, University of Padua, Padua, Italy

${ }^{2}$ Division of Gastroenterology, Department of Internal Medicine, University of Pisa, Pisa, Italy

${ }^{3}$ Division of Gastroenterology, Department of Internal Medicine, University of Genoa, Genoa, Italy

${ }^{4}$ Division of Pharmacology and Chemotherapy, University of Pisa, Pisa, Italy

\section{Corresponding author:}

Edoardo Savarino, MD

Division of Gastroenterology, Department of Surgery, Oncology and Gastroenterology, University of Padua, Padua, Italy

Via Giustiniani 2

35128 - Padova - Italy

Phone: +390498217749

Fax: +390103538956 


\begin{abstract}
Introduction: About $30-40 \%$ of GERD patients report an inadequate response to proton pump inhibitors (PPIs) due to their suboptimal pharmacological profiles. Recently, a new synthesized PCABs, vonoprazan, showed higher suppression of gastric acid secretion as compared to lansoprazole.

Areas covered: This review provides an update on the pharmacokinetic properties of vonoprazan and their correlates with pharmacodynamics; preliminary data on the therapeutic efficacy of vonoprazan as compared to lansoprazole in GERD patients
\end{abstract}

Expert opinion: At variance from all available PPIs, vonoprazan acts directly on $\mathrm{H}+, \mathrm{K}+$-ATPase irrespectively of its activity, providing a fast onset of action without requiring acid activation and specific administration timing. Clinical and pharmacological investigations have confirmed a more rapid, potent and prolonged inhibition of acid secretion, including a better nighttime acid control, and a less antisecretory variability, as compared with PPIs. Preliminary data in patients with erosive esophagitis (EE) have shown the non-inferiority of vonoprazan to lansoprazole in terms of symptom relief and healing rate. Since these pharmacokinetic advantages, it is expected that it will have a significant favorable impact on GERD management. However, the clinical use of vonoprazan raises also some issues about its efficacy and safety in the long-term that deserve verification and careful investigation.

Keywords: Vonoprazan, Gastro-esophageal Reflux Disease, GERD, Pharmacokinetics, P-CABs 


\section{Article highlights box}

- About $30-40 \%$ of gastro-esophageal reflux disease (GERD) patients report an inadequate response to proton pump inhibitors drugs due to their suboptimal pharmacological profiles.

- Recently, a new synthesized Potassium-competitive acid blocker, Vonoprazan, has been developed by Takeda Pharmaceuticals with preliminary data showing higher suppression of gastric acid secretion and similar efficacy as well as safety as compared to lansoprazole in GERD patients

- At variance from all the available PPIs, vonoprazan acts directly on $\mathrm{H}+, \mathrm{K}+$-ATPase irrespectively of their activity, providing a fast onset of action without requiring acid activation and specific timing with respect to food intake,

- Clinical and pharmacological investigations have confirmed a more rapid, potent and prolonged inhibition of acid secretion by vonoprazan, including a better nighttime acid control, as compared with PPIs, with a less antisecretory variability and a lower risk of drug interactions among patients.

- First clinical data in patients with erosive esophagitis (EE) have shown that the healing rate after two weeks of treatment with vonoprazan was higher than lansoprazole, with the proportion of patients with healed EE displaying a higher trend in the vonoprazan group, in patients with more severe esophagitis.

- Further studies investigating the efficacy and safety in the long-term of vonoprazan in patients with acid-related disorders are required 


\section{Introduction}

Gastroesophageal reflux disease (GERD) develops when the reflux of gastric contents into the esophagus causes troublesome symptoms and/or complications.[1-3] Population-based studies suggest that GERD prevalence is increasing worldwide, with relevant impact on quality of life and health care costs.[4]

The pathogenesis of GERD is multifactorial in nature, mainly involving dysfunctions of the esophagogastric junction, ineffective esophageal acid and bolus clearance, impaired mucosal defensive factors, and esophageal hypersensitivity.[5] Moreover, characteristics and components of the refluxate play a pivotal role in the pathological consequences of GERD. [6]

To date, acid suppression therapy represents the mainstay of GERD treatment.[7, 8] Pathophysiological studies have indeed shown that the vast majority of GERD symptoms are associated with acid reflux episodes,[9] and that the exposure of esophageal mucosa to acid increases progressively in parallel with the increasing severity of GERD presentation.[10, 11]

The breakthrough in the treatment of acid-related diseases was the discovery of histamine-2receptor antagonists (H2RAs) in the mid-1970s.[12, 13] However, these drugs, which act through the blockade of only one of the three main pathways regulating gastric acid secretion, displayed soon a number of significant limitations, including a relatively short duration of action, an incomplete inhibition of post-prandial gastric acid secretion, a decline in the antisecretory effect (i.e., tolerance), and a post-treatment rebound acid hypersecretion.[14-17]

The identification of the proton pump $(\mathrm{H}+, \mathrm{K}+$-ATPase $)$ as the final step of gastric acid secretion and the development of its blockers (proton pump inhibitors, PPIs) led to a significant improvement in the management of acid-related disorders.[18] Indeed, PPIs provide a greater and longer lasting effect on acid suppression than H2RAs,[19] with a more potent symptomatic relief and esophagitis healing in high proportions of GERD patients.[19-21] Furthermore, PPIs control both basal and food-stimulated acid secretion, and they are not subjected to development of tolerance.[17, 18, 22] PPIs represent currently the mainstay of GERD treatment.[7] However, failure or incomplete 
response to these drugs has been reported from 10 to $40 \%$ of GERD patients.[23, 24] Although the causes of refractoriness to PPIs are multiple in nature,[25-28] they can be partly linked to shortcomings and limitations pertaining to PPIs, as discussed in detail below.

Overall, the above considerations underscore the need for new options in the therapeutic management of GERD. In this context, current research is being focused on potassium-competitive acid blockers (P-CABs). Among them, a novel P-CAB, vonoprazan, designated also as TAK-438 (Box 1), has been developed.[29, 30] In the present article, the available information on vonoprazan have been reviewed to provide an appraisal of its pharmacokinetic profile, antisecretory activity and efficacy in GERD patients. Special attention has been paid to comparative data with lansoprazole in terms of acid inhibition, relief of GERD symptoms and mucosal healing.

\section{Role of PPIs in GERD: main determinants of their antisecretory effect and limitations}

PPIs represent the first choice medical treatment for GERD. In this setting, the main therapeutic indications for PPI use include the induction of symptom remission and esophagitis healing, as well as the maintenance of symptom control and prevention of esophagitis recurrence.[7, 31-33]

PPIs are targeted at selectively and irreversibly blocking the gastric $\mathrm{H}+, \mathrm{K}+$-ATPase, which is the final step in acid secretion. Of note, their inhibitory action can be exerted only on active protonic pumps, that is when, under appropriate stimuli, $\mathrm{H}^{+}, \mathrm{K}+-\mathrm{ATPase}$ translocates to the apical membrane of parietal cells and operates to decrease the $\mathrm{pH}$ of secretory canalicula to approximately 1.0.[34, 35] Under resting conditions, parietal cells can initially escape the acid inhibitory action of PPIs, and therefore a full antisecretory effect is usually obtained after administration of repeated doses (steady-state inhibition is achieved within 3 to 5 days).[36, 37]

With regard for their mechanism of action, PPIs are weak base prodrugs (substituted pyridylmethylsulfinyl benzimidazoles with $\mathrm{pKa}$ values ranging from 3.8 to 4.5 ) that, upon oral administration enter the systemic blood circulation and accumulate in the strong acid microenvironment of secretory canalicula, where they undergo activation through an acid-catalyzed 
conversion into a thiophylic cyclic sulfonamide that is a highly reactive molecular species.[18] This compound then inhibits $\mathrm{H}+-\mathrm{K}+-\mathrm{ATPase}$ by means of covalent disulfide bonds with cysteine residuals exposed in the luminal domain of the proton pump.[34] Since PPIs are acid-labile compounds and proton pump blockade requires their acid-dependent activation within the secretory canalicula of parietal cells, these drugs must be administered orally as gastroprotected formulations, to protect them from acid degradation within the gastric lumen.[36, 37]

When considering PPI pharmacokinetics, there is evidence that the area under the plasma concentration-time curve (AUC) represents a major determinant of the magnitude of the acid inhibitory effect, and that the plasma elimination half-life $\left(t^{1} / 2\right)$ is a major determinant of the effect duration. Despite their short plasma $\mathrm{t}^{1} \frac{2}{2}$ of $60-90$ minutes, the covalent binding of PPIs with proton pumps accounts for their long-lasting acid inhibitory effect. However, gastric proton pumps have a half-life of 50 hours and about one third of them are replaced by new biosynthesis every day. Thus, the new proton pumps synthetized at night-time cannot be exposed to PPIs, even when these drugs are administered twice daily, with a consequent persistence of low volume acid secretion (nocturnal acid breakthrough).[37] Most PPIs are known also to undergo hepatic metabolism by the CYP system. In this regard, CYP2C19 gene polymorphisms have been shown to influence the pharmacokinetics of some PPIs, thus generating relevant inter-individual variability of their pharmacodynamics and clinical activity. [38, 39]

PPIs can interfere with other drugs because of their inhibitory effect on gastric acid secretion or their inhibition of enzymes involved in drug metabolism.[40]

With respect to safety, most studies on short- and long-term PPI use have provided reassuring data.[41] Nevertheless, adverse effects associated with long-term PPI use deserve careful consideration.[42]

Overall, current PPIs cannot be considered ideal drugs for GERD due to their limitations, including: 1) lack of targeting of key GERD pathophysiological mechanisms; 2) suboptimal efficacy of symptom control; 3) suboptimal pharmacological profiles in terms of intake at appropriate time 
relative to meals, delay in effect onset, low bioavailability, variable liver metabolism, and nocturnal acid breakthrough.[43-45]

To date, new pharmaceutical strategies to overcome PPI limitations in GERD therapy have been focused on: 1) new formulations of current PPIs; 2) new PPIs (i.e. tenatoprazole, ilaprazole); 3) new proton pump blockers endowed with different mechanisms of action than benzimidazole PPIs (PCABs).[43, 46]

\section{PCABs}

P-CABs comprise currently a heterogeneous group of molecules able to inhibit selectively the gastric $\mathrm{H}+, \mathrm{K}+$-ATPase by a specific $\mathrm{K}+$-competitive reversible mechanism.[47, 48]

P-CABs are weak bases endowed with a higher pKa than PPIs. Accordingly, P-CABs are highly concentrated in the acidic secretory canalicula of parietal cells, and are instantly protonated when exposed to the acidic environment. Once in the protonated form, P-CABs are thought to bind and inhibit the $\mathrm{K}+$ site of $\mathrm{H}+, \mathrm{K}+$-ATPase.[49] As anticipated above, PPIs are prodrugs that exert their inhibitory activity after undergoing an acid-catalyzed conversion to a reactive species. At variance, P-CABs do not require any molecular rearrangement under acidic conditions, thus implying that they are more stable and not subjected to degradation at the $\mathrm{pH}$ levels achievable in the gastric lumen. As a result, $\mathrm{P}-\mathrm{CABs}$ can be administered by oral route without requiring gastroprotective formulations. Moreover, PCABs achieve rapidly peak plasma concentrations after oral administration, their control on gastric $\mathrm{pH}$ throughout the $24-\mathrm{h}$ period is more stable than PPIs, and the duration of their antisecretory effect is related to their plasma $t^{1} \frac{1}{2}$.[50]

P-CABs have been shown to inhibit the $\mathrm{K}+$-stimulated dephosphorylation of $\mathrm{H}+, \mathrm{K}+$-ATPase by a competitive displacement of $\mathrm{K}+$ from the phosphorylated proton pump.[51] This mechanism allows P-CABs to rapidly block the $\mathrm{H}+, \mathrm{K}+$-ATPase in its mid-cycle, without requiring proton pump activation, thus resulting in a more rapid acid inhibition and elevation of gastric $\mathrm{pH}$ to higher levels than PPIs.[52] In line with this expectation, PCABs displayed a faster onset of acid inhibitory 
action, when compared to PPIs, reaching a full antisecretory effect since the first dose, within the first day.[53, 54]

The imidazopyridine SCH28080 was the first compound developed as a P-CAB. It was shown to be a pure $\mathrm{K}+$ competitive inhibitor of the gastric proton pump with a $\mathrm{pKa}$ of 5.5. Accordingly, SCH28080 displayed an efficient acid inhibitory action and a selective accumulation in the acid compartment of parietal cells. Nevertheless, it was never marketed owing to its hepatoxicity.[55] Afterwards, other similar drugs have been synthesized and evaluated, such as revaprazan (a pyrimidine derivative) and AZD0865 (another imidazopyridine compound). However, despite a good acid inhibitory activity, these drugs were limited by their rapid dissociation rate from the proton pump that was responsible for a short duration of acid inhibition and, consequently, an incomplete control of gastric acid secretion over $24-\mathrm{h}$, particularly at night-time, when administered on a once-a-day basis.[56, 57]

Acknowledging the benefits of P-CABs, as compared to PPIs, Takeda Pharmaceutical Company Limited (Japan) recently developed a novel and potent compound (vonoprazan), endowed with a different chemical structure from previous P-CABs, with the goal of overcoming the shortcomings of its predecessors.

\section{Vonoprazan}

Vonoprazan is a pyrrole derivative, 1-[5-(2-fluorophenyl)-1-(pyridin-3-ylsulfonyl)-1H-pyrrol-3-yl]$\mathrm{N}$-methylmethanamine monofumarate (designated also as TAK-438), characterized by a slightly slower onset of action, as compared to previous P-CABs, while displaying higher affinity for gastric $\mathrm{H}+, \mathrm{K}+-\mathrm{ATPase}$ and a slower dissociation rate.[58]

\subsection{Pharmacodynamics}

\subsubsection{Studies in preclinical models}


Vonoprazan is a potent $\mathrm{K}+$-competitive inhibitor of the gastric $\mathrm{H}+, \mathrm{K}+$-ATPase, with an inhibitory constant (Ki) of $10 \mathrm{nM}$ at $\mathrm{pH} 7$, and $3 \mathrm{nM}$ at $\mathrm{pH}$ 6.5.[58, 59] When compared with SCH28080 and lansoprazole, under in vitro weakly acidic conditions ( $\mathrm{pH}$ 6.5), all three compounds inhibited the activity of gastric $\mathrm{H}+, \mathrm{K}+$-ATPase in a concentration-dependent manner. However, the inhibitory activity of vonoprazan was the most potent. Moreover, under neutral conditions ( $\mathrm{pH} 7.5$ ), the inhibitory activity of SCH28080 and lansoprazole decreased, while that of vonoprazan remained almost the same as that recorded at $\mathrm{pH}$ 6.5.[59] Indeed, the $\mathrm{pKa}$ value of vonoprazan (9.37) is higher than that of previous P-CABs, and therefore it is expected to be protonated instantly and exert a potent inhibitory activity on gastric proton pumps even in a neutral environment.[59]

The binding rate of vonoprazan in vitro reaches the plateau of inhibition within $200 \mathrm{~s}$. However, such a slow action onset does not appear to affect its efficacy on diurnal $\mathrm{pH}$ control in vivo.[58, 59] As an additional advantage, vonoprazan has a slower dissociation rate from proton pumps than other P-CABs that translates into a longer duration of its acid inhibitory activity. Shin et al.[58] observed that the dissociation half-life of vonoprazan was inversely related with the concentration of potassium chloride $(\mathrm{KCl})$ in the incubation medium $(12.5 \mathrm{~h}, 7.5 \mathrm{~h}$ and $3 \mathrm{~h}$ at $10 \mathrm{mM}, 20 \mathrm{mM}$ and $30 \mathrm{mM} \mathrm{KCl}$, respectively).[58] At higher concentrations of vonoprazan, higher concentrations of $\mathrm{KCl}$ were required to maintain the same dissociation rate. Of note, Keeling et al.[60] reported that about $60 \%$ of SCH28080 dissociated from proton pumps within $2 \mathrm{~min}$ in $10 \mathrm{mM} \mathrm{KCl}$. As shown by Shin et al.,[58] in the presence of acidic media the inhibitory effect of vonoprazan on proton pumps is less sensitive to $\mathrm{KCl}$, since at lower $\mathrm{pH}$ there is a predominance of the protonated form of vonoprazan, which is endowed with a stronger binding affinity, and thus it is less influenced by $\mathrm{KCl}$ concentration. According to the hypothesis that $\mathrm{H}+, \mathrm{K}+$-ATPase is commonly exposed on the apical membrane of parietal cells at $15 \mathrm{mM} \mathrm{KCl}$ under conditions of stimulated gastric acid secretion, the expected in vivo dissociation rate of vonoprazan should be longer than $7.5 \mathrm{~h}$. Therefore, when translating these in vitro findings to the clinical setting, a stable acid inhibition is expected to occur after a single administration of vonoprazan.[58] To describe whether vonoprazan binding to gastric 
$\mathrm{H}+, \mathrm{K}+-\mathrm{ATPase}$ was covalent or reversible in nature, Shin et al.[58] exposed the gastric pump to vonoprazan and, subsequently, to [14C]-labeled vonoprazan. After precipitation of [14C]vonoprazan-bound enzyme, $99 \%$ of [14C]vonoprazan and only $1 \%$ of vonoprazan were found in the supernatant, thus substantiating the reversible inhibition of the $\mathrm{H}+, \mathrm{K}+$-ATPase.[58] Matsukawa et al.[61] investigated the accumulation of vonoprazan in the canalicula of parietal cells by comparison with lansoprazole in primary cultured rabbit gastric glands. As expected, lansoprazole accumulation was dependent on acid secretion and higher under the active than under the resting conditions of proton pumps. Under these conditions, both lansoprazole and vonoprazan inhibited acid secretion in a concentration-dependent manner. However, vonoprazan was confirmed to be a more potent inhibitor than lansoprazole (IC50 0.30 vs $0.76 \mu \mathrm{M}$ ). Moreover, vonoprazan accumulated in both resting and stimulated glands more than lansoprazole, and its clearance was slower. In particular, when drug clearance was evaluated by means of [14C]-labeled compounds, the radioactive ratio of lansoprazole at 24 -h over the baseline was higher than that of vonoprazan. Such a result might be explained by the covalent binding of lansoprazole against the reversible inhibition operated by P-CABs. However, the amount of vonoprazan retention at 24-h was greater than that of lansoprazole. Of note, in this study the use of cultured gastric glands allowed to measure the effective accumulation and clearance of test drugs for a longer period, as compared to data obtained from acutely isolated gastric glands. Based on these data, it could be hypothesized that vonoprazan is able to generate a stronger and more prolonged inhibition, owing to its large accumulation and slow clearance in gastric glands.[61]

With regard for selectivity, vonoprazan has been shown to not inhibit $\mathrm{Na}+, \mathrm{K}+$-ATPase due to its high affinity for $\mathrm{H}+, \mathrm{K}+-\mathrm{ATPase}$. By contrast, lansoprazole can exert a minimal inhibitory action on $\mathrm{Na}+, \mathrm{K}+$-ATPase.[58, 59] To examine further the binding selectivity of vonoprazan for the gastric $\mathrm{H}+, \mathrm{K}+-\mathrm{ATPase}$ and its slow dissociation rate, Scott et al.[62] performed an autoradiography of vonoprazan binding and a molecular modelling of the proton pump. They observed that, at variance from omeprazole, labelling of rabbit gastric glands with [14C]vonoprazan did not change under 
resting or stimulated conditions. Vonoprazan binding was therefore independent of acid secretion, while acid activation is required for PPIs. Moreover, the selective labelling of parietal cells, regardless of the active or inactive state of $\mathrm{H}+, \mathrm{K}+$-ATPase, showed that it is the high affinity of vonoprazan that determines its binding selectivity, and not the $\mathrm{pH}$ within the canalicular compartment.[62]

In their study, Scott et al.[62] tried to identify the mechanism responsible for the high affinity of vonoprazan by a modelling of drug binding to the $\mathrm{H}+, \mathrm{K}+$-ATPase. The kinetic analysis of wild type gastric proton pumps confirmed a $\mathrm{Ki}$ of $3.0 \mathrm{nM} / \mathrm{L}$ for vonoprazan, while the abrupt loss of affinity for the mutant try799ser indicated the significance of the phenyl side chain for the binding of vonoprazan. In addition, the authors observed that a higher total surface area and a higher percentage of vonoprazan was engaged into the binding site, as compared to SCH28080, showing that vonoprazan fills the luminal vestibular space of the proton pump more efficiently than SCH28080.[62] This observation might explain the slower dissociation rate of vonoprazan. Use of mutant models allowed to identify also some subtle conformational differences that might explain the higher affinity of vonoprazan for the gastric $\mathrm{H}+, \mathrm{K}+$-ATPase, as compared to $\mathrm{SCH} 28080$, and the high selectivity of vonoprazan for $\mathrm{H}+, \mathrm{K}+$-ATPase over the ubiquitous $\mathrm{Na}+, \mathrm{K}+$-ATPase.[58, 62] Overall, based on the above observations, a pKa of 9.3 , a $\mathrm{Ki}$ of $3.0 \mathrm{nM} / \mathrm{L}$ and the wide binding surface of vonoprazan appear to be the key points that would explain its more potent and longer lasting antisecretory action as compared with other P-CABs.

In vivo preclinical studies have supported the view that vonoprazan exerts a more potent and longerlasting antisecretory activity than previous P-CABs and PPIs. Hori et al.[59] assessed the effects of vonoprazan on acid secretion and $\mathrm{pH}$ of gastric perfusate in rats, as compared to lansoprazole and SCH28080. When administered to rats, vonoprazan showed maximal acid inhibitory activity at the dose of $4 \mathrm{mg} / \mathrm{kg}$, while lansoprazole showed potent, but incomplete, inhibition of acid secretion at 4 or $8 \mathrm{mg} / \mathrm{kg}$. This finding was confirmed also under stimulation of gastric acid secretion with 2deoxy-D-glucose or histamine.[59] Moreover, vonoprazan increased the $\mathrm{pH}$ of gastric perfusate to a 
higher value than lansoprazole or SCH28080, and the effect of vonoprazan was more sustained than that of lansoprazole or SCH28080.[59] Similar results have been obtained in a subsequent study by the same authors, who measured the $\mathrm{pH}$ of gastric perfusate in rats and dogs after administration of vonoprazan or lansoprazole. In this study, at variance from lansoprazole, the increase in $\mathrm{pH}$ by vonoprazan was unaffected by subsequent stimulation of gastric acid secretion with histamine.[63] In line with these findings, Arikawa et al.[64] confirmed a more potent antisecretory activity of vonoprazan on histamine-induced acid secretion in rats and dogs, as compared with lansoprazole.

\subsubsection{Studies in humans}

Studies on healthy volunteers have shown rapid, marked, and sustained inhibition of gastric acid secretion with single oral doses of vonoprazan.[29, 65] In two phase I, randomized, double-blind, placebo-controlled, single rising-dose studies, performed in Japan and United Kingdom (UK), healthy male subjects received a single dose of vonoprazan (1-120 mg in Japan, and 1-40 $\mathrm{mg}$ in the UK). Acid inhibition showed a rapid onset, being dose-dependent and similar in both studies. The 24-h intragastric $\mathrm{pH} \geq 4$ holding time ratio (HTR) with $40 \mathrm{mg}$ of vonoprazan was $92 \%$ in Japan and $87 \%$ in the UK.[65] The same authors performed two additional phase I studies on healthy male subjects in Japan and UK, where vonoprazan was administered at multiple rising oral doses, ranging from 10 to $40 \mathrm{mg}$, once daily for 1 week, showing similar pharmacodynamics as the above mentioned studies.[29] In particular, the intragastric $\mathrm{pH}$ HTRs on day 7 displayed a good $\mathrm{pH}$ control throughout the 24-h dosing interval ( $\mathrm{pH}>4$ HTR $>83 \%$ ), including the night-time period (pH>4 HTR $\geq 73 \%$ ), at the clinically recommended dose of $20 \mathrm{mg}$, with even a greater rate of acid inhibition observed with daily doses $>20 \mathrm{mg}$. Overall, the authors concluded that $20 \mathrm{mg} /$ day was efficient, with a fast onset of action, offering a good acid control throughout 24-h and at nighttime.[29]

Two comparative studies of vonoprazan with PPIs in healthy volunteers carrying the CYP2C19 extensive metabolizer genotype have been also performed.[66] Firstly, in a randomized, open-label, 
two-period cross-over study, vonoprazan $20 \mathrm{mg}$ and esomeprazole $20 \mathrm{mg}$ or rabeprazole $10 \mathrm{mg}$ were orally administered daily for 7 days. Under these conditions, vonoprazan exerted a more rapid and sustained acid-inhibitory effect. Indeed, acid-inhibition was evident after the first administration of vonoprazan, and it was sustained over 24-h during 7 days.[66] More recently, in a randomized, two-period cross-over study, vonoprazan $20 \mathrm{mg}$ twice daily, vonoprazan $20 \mathrm{mg}$ daily, esomeprazole $20 \mathrm{mg}$ twice daily and esomeprazole $20 \mathrm{mg}$ daily were orally administered daily for 7 days in 28 healthy volunteers [7 CYP2C19 poor metabolisers (PMs), 11 intermediate metabolisers (IMs) and 10 rapid metabolisers (RMs)]. Vonoprazan twice daily potently suppressed acid for $24 \mathrm{~h}$, and was significantly superior to other regimens irrespective of CYP2C19 genotype. Vonoprazan daily was equivalent to esomeprazole twice daily in IMs and PMs, but superior in RMs. Thus, CYP2C19 genotype-dependent differences were observed in esomeprazole daily but not in vonoprazan twice daily or daily.[67]

\subsection{Pharmacokinetics}

The chemical structure of vonoprazan is characterized by a point-positive charge (pKa 9.06) that favors a greater accumulation in parietal cells as compared with previous P-CABs.[58] The duration of the antisecretory effect of vonoprazan is also dependent on its $t^{1 / 2}$, which is 7.5 hours (after oral administration in $20 \mathrm{mM}$ of $\mathrm{KCl}$ at $\mathrm{pH} 7)$.[58]

In the two phase Nstudies performed in Japan and UK on healthy male subjects, plasma concentration-time profiles of vonoprazan at all dose levels showed a pattern of rapid absorption (median Tmax up to $2 \mathrm{~h}$ ) under fasting conditions. The estimated mean elimination half-life was up to $9 \mathrm{~h}$. The systemic exposure (i.e., maximum observed concentration, Cmax, and bioavailability, AUC) was slightly greater than dose proportional. The urinary excretion of unchanged vonoprazan over 24-h was low after all the tested doses (about 1-9\%). Overall, no clear difference in vonoprazan pharmacokinetics was observed between Japanese and non-Japanese subjects.[65] 
Similar results were obtained in subsequent two phase I studies on healthy male volunteers performed by the same authors.[29]

Of note, vonoprazan metabolism displays a limited influence by CYP polymorphisms.[64] In particular, both in healthy male volunteers and GERD patients, it has been shown that the pharmacokinetics and pharmacodynamics of vonoprazan were not affected by the CYP2019 genotype.[29, 65, 66, 68]

\subsection{Efficacy in GERD}

Ashida et al.[68, 69] performed two phase II, multicentre, randomised, double-blind, parallel-group studies in Japanese patients with endoscopically confirmed erosive esophagitis. The first study was designed to evaluate the efficacy of once-daily vonoprazan at 5, 10, 20 or $40 \mathrm{mg}$ versus once-daily $30 \mathrm{mg}$ lansoprazole in healing erosive esophagitis at week 4, as assessed by endoscopy.[69] They observed that vonoprazan was effective and non-inferior to lansoprazole in healing esophagitis at all the tested doses. Of interest, the healing rate after two weeks of treatment with vonoprazan $20 \mathrm{mg}$ was higher than with lansoprazole $30 \mathrm{mg}$. Moreover, although differences in the proportion of healed erosive esophagitis did not reach statistical significance, $20 \mathrm{mg}$ or higher doses of vonoprazan resulted to be highly effective in patients with severe esophagitis (grades C/D). In conclusion, the authors recommended $20 \mathrm{mg}$ /day of vonoprazan as the most suitable clinical dose for the management of erosive esophagitis.[69]

In the second study, Ashida et al.[68] aimed at verifying the non-inferiority of vonoprazan 20 mg/day versus lansoprazole $30 \mathrm{mg} /$ day in patients with erosive esophagitis, and then to establish long-term efficacy of vonoprazan as maintenance therapy. In this study, vonoprazan and lansoprazole were equally effective at 8 weeks. However, there was a much higher percentage of patients healed at week 2 in the vonoprazan group (90.7\% versus $81.9 \%$; 95\% CI: $2.105-15.448$; P $<0.0001)$. Moreover, the analysis of patient subgroups showed that the proportion of patients with healed erosive esophagitis tended to be higher in the vonoprazan group, as compared with 
lansoprazole, in patients with more severe esophagitis (grades C/D) as well as in those classified as CYP2C19 extensive metabolisers. Subsequently, patients, who had been healed at 8 weeks, were randomized to receive vonoprazan 10 or $20 \mathrm{mg} /$ day as maintenance therapy up to 52 weeks. Over this time, the recurrence rate of erosive esophagitis was low $(<10 \%)$.[68] Overall, these results suggest a stronger and faster clinical effect with vonoprazan, which presumably results from its rapid and strong suppression of gastric acid secretion.

\subsection{Safety profile}

The safety and tolerability of vonoprazan have been investigated in the above mentioned phase I studies, performed by Sakurai et al.[65] on healthy male subjects volunteers. The drug was administered according to a single-dose escalation scheme aimed at finding up the maximum tolerated dose or the maximum AUC. In this setting, vonoprazan was safe and well tolerated at all tested doses, without relevant adverse events in the Japanese subjects and only 12 treatmentemergent adverse events (TEAEs) in 10 subjects of the UK study. There were no serious adverse events in either study.[65] In two subsequent phase I studies conducted by the same authors on healthy male subjects, vonoprazan was administered at multiple rising oral doses, ranging from 10 to $40 \mathrm{mg}$, once daily for 1 week, and all doses were found to be safe and well tolerated.[29] Likewise, in a further randomized, open-label, two-period cross-over study, vonoprazan $20 \mathrm{mg}$, administered daily for 7 days by oral route, was well tolerated.[66]

In a randomized, dose-ranging study, the safety profile of vonoprazan was evaluated in patients with erosive esophagitis at doses of 5, 10, 20 and $40 \mathrm{mg}$ once daily for 8 weeks. Overall, vonoprazan was safe and well tolerated at all the investigated doses.[69] Subsequently, the same authors investigated the long-term safety of vonoprazan in a similar study in patients with erosive esophagitis. In this setting, there were no new safety signals and no significant changes in laboratory values, vital signs or electrocardiogram findings, other than an increase in gastrin levels. Accordingly, it was concluded that vonoprazan at the doses of 10 and $20 \mathrm{mg}$ once daily, as maintenance therapy for 52 
weeks, was safe and well-tolerated.[68] Of note, at variance with previous P-CABs, the vonoprazan structure lacks the imidazopyridine ring, which was responsible for hepatotoxicity.

\section{Conclusion}

Acid suppression with PPIs represents the mainstay of GERD medical treatment. However, despite the well-documented clinical efficacy and safety of PPIs, about $20-40 \%$ of GERD patients display an inadequate response to these drugs, thus underscoring the need for new drugs able to achieve a more potent and long-lasting inhibition of gastric acid secretion. In this context, vonoprazan, a novel pyrrole derivative $\mathrm{P}-\mathrm{CAB}$, has been developed as a potential alternative to PPIs for the management of acid-related disorders, including GERD. This drug has been shown to compete with $\mathrm{K}+$ and to induce a selective and reversible inhibition of $\mathrm{H}+-\mathrm{K}+$-ATPase in a dose-dependent manner.

Studies in preclinical models and humans have documented the ability of vonoprazan of inhibiting the gastric proton pump more effectively than previous P-CABs and PPIs. In particular, vonoprazan is endowed with a pKa of 9.3, which is higher than that of previous P-CABs and PPIs. Therefore, its pharmacodynamic activity is not affected by higher values of $\mathrm{pH}$ and the compound is resistant to strong acidity. Once in an acidic environment, vonoprazan is instantly protonated. It is then highly concentrated and remains in its protonated form within the secretory canalicula of gastric parietal cells, where it binds and inhibits the proton pumps. Of note, at variance from PPIs, the acid inhibitory effect of vonoprazan does not require proton pump activation and it is unaffected by the gastric secretory activity, thus resulting in a more rapid acid inhibition and elevation of gastric $\mathrm{pH}$ than PPIs. Consistently with these patterns, vonoprazan has been shown to exert a more potent and longer-lasting inhibitory effect on gastric acid secretion, as compared with PPIs and previous PCABs. The specific advantages of vonoprazan, as compared to previous P-CABs, include a slower dissociation rate from the $\mathrm{H}+, \mathrm{K}+$-ATPase, that accounts for a longer duration of action, and the lack of hepatotoxicity that has precluded the clinical use of other PCABs. Molecular modellings of 
vonoprazan binding to $\mathrm{H}+, \mathrm{K}+$-ATPase have suggested that its specific conformational features represent a possible explanation whereby its exit from the binding site is more hindered than previous P-CABs. As a result of these properties, human studies have shown that vonoprazan can exert its full antisecretory activity since the first dose and provide sustained control of acid secretion, including the nocturnal period. Furthermore, at variance with most of current PPIs, the metabolic disposition of vonoprazan is not significantly influenced by CYP2C19 genetic polymorphism, thus resulting in a low interindividual variability. Whether these favorable pharmacological properties will translate into greater clinical benefits in GERD patients, as compared with current PPI therapy, has yet to be conclusively established.

\section{Expert opinion}

PPIs have a number of shortcomings, which arise from their pharmacological properties. Firstly, PPIs display a large interindividual variability in their own disposition, which depends on both genetic and non-genetic factors, and therefore the degree of acid achieved by a standard, once-daily dose of any available PPI, is highly variable over the 24-h, with possible persistence of nocturnal acidity. In addition, all available PPIs need several days to achieve optimal efficacy. Furthermore, a major drawback of PPIs is the requirement for acid secretion within the secretory canalicula of parietal cells to promote the accumulation of the prodrug and the conversion into its active form.

Consistently with the lack of an ideal drug, there are still some unmet clinical needs in GERD that deserve consideration and await for adequate solutions. These include: the management of patients with reflux symptoms who do not respond adequately to PPI therapy; a faster onset of symptom relief; the optimization of acid inhibition with regard for the daily time course of gastric $\mathrm{pH}$ control, particularly over the night. In this context, an innovative approach has been the development of a new class of $\mathrm{H}+, \mathrm{K}+$-ATPase inhibitors, designated as PCABs, among which vonoprazan has been currently approved in Japan for the treatment of acid-related diseases, including GERD, erosive esophagitis, gastric and duodenal ulcer, and Helicobacter pylori eradication. 
Vonoprazan inhibits $\mathrm{H}+, \mathrm{K}+$-ATPase in a reversible and $\mathrm{K}+$-competitive manner, providing a fast onset of action. At variance from all the available PPIs, vonoprazan acts directly on $\mathrm{H}+, \mathrm{K}+-$ ATPase, without requiring acid activation. Since the onset of acid inhibition occurs irrespectively of the activity of proton pumps, vonoprazan does not require specific timing with respect to food intake. In addition, as vonoprazan is stable in acidic environments, no gastro-protective coating is required. In line with these pharmacological properties, experimental studies and clinical pharmacological investigations in healthy volunteers have confirmed a more rapid, potent and prolonged inhibition of acid secretion by vonoprazan, including a better nighttime acid control, as compared with PPIs. At last, vonoprazan is not metabolized by CYP2C19, allowing for less antisecretory variability and a lower risk of drug interactions among patients, particularly those receiving poly-drug therapies.

The results of trials in Japanese patients with erosive esophagitis have shown that the healing rate after two weeks of treatment with vonoprazan was higher than lansoprazole, with the proportion of patients with healed erosive esophagitis displaying a higher trend in the vonoprazan group, in patients with more severe esophagitis.

Since vonoprazan provides a more effective control of intragastric $\mathrm{pH}$, it is expected that it will have a significant favorable impact on GERD management. In particular, patients complaining of persistent symptoms late in the day or at night will be among the expected beneficiaries of the improved pharmacological properties of vonoprazan. The fast onset of action, with a full acid inhibition since the first dose, is expected also to be clinically relevant, making vonoprazan suitable for on-demand maintenance therapy.

Besides a number of expected benefits, the clinical use of vonoprazan raises also some issues that deserve verification and careful investigations. First, given the limited clinical data available on vonoprazan, additional and more extensive randomized controlled clinical trials are urgently required to confirm its actual advantages in GERD patients, with and without erosive esophagitis. In particular, comparative clinical studies of vonoprazan versus second generation PPis (rabeprazole 
and esomeprazole), assessing the potency and time course of gastric $\mathrm{pH}$ control, as well as the suitability of vonoprazan for both short- and long-term maintenance of GERD, including healing of erosive esophagitis and symptom relief, are warranted. Second, special attention should be paid to randomized controlled trials evaluating the potential effectiveness of vonoprazan in patients with refractoriness to therapy with current PPIs. Indeed, considering that not all GERD patients will require vonoprazan as first line therapy, we deem of interest to implement studies on targeted patient populations, who could take particular advance from a more-potent acid inhibition (i.e., patients with atypical GERD symptoms). Third, other areas deserving attention include the potential interactions of vonoprazan with other drugs, as well as the overall safety profile of vonoprazan given over long-term treatment periods.

\section{Drug Summary Box}

\begin{tabular}{|l|l|}
\hline Drug name & $\begin{array}{l}\text { Aonoprazan fumarate } \\
\text { esophageal reflux disease and peptic } \\
\text { ulcer) } \\
\text { Adjunct to Helicobacter pylori } \\
\text { eradication }\end{array}$ \\
\hline Indication & $\begin{array}{l}\text { Potassium-competitive acid blocker } \\
\text { (P-CAB) }\end{array}$ \\
\hline Pharmacology description & Oral \\
\hline Chemical structure & \\
\hline Pivotal trial(s) & \\
\hline
\end{tabular}




\section{Authors contributions}

Edoardo Savarino, Irene Martinucci, Manuele Furnari, Nicola de Bortoli,: design of the study, data collection, writing of the manuscript, approving final version.

Gaia Pellegatta, Chiara Romana, Alessandro Moscatelli, Giorgia Bodini, Elisa Marabotto: data collection, writing of the manuscript, approving final version

Corrado Blandizzi, Vincenzo Savarino: writing of the manuscript, manuscript revision, approving final version.

\section{Funding}

This paper was not funded

\section{Declaration of Interest}

The authors have no relevant affiliations or financial inyolvement with any organization or entity with a financial interest in or financial conflict with the subject matter or materials discussed in the manuscript. This includes employment, consultancies, honoraria, stock ownership or options, expert testimony, grants or patents received or pending, or royalties.

\section{Bibliography}

Papers of special note have been highlighted as either of interest $(\bullet)$ or of considerable interest $(\bullet \bullet)$ to readers.

1 Vakil N, van Zanten SV, Kahrilas P, Dent J, Jones R, Global Consensus G. The Montreal definition and classification of gastroesophageal reflux disease: a global evidence-based consensus. The American journal of gastroenterology 2006 Aug;101(8):1900-20; quiz 43.

2. de Bortoli N, Nacci A, Savarino E, Martinucci I, Bellini M, Fattori B, et al. How many cases of laryngopharyngeal reflux suspected by laryngoscopy are gastroesophageal reflux disease-related? World journal of gastroenterology : WJG 2012 Aug 28;18(32):4363-70.

3. Richter JE, Penagini R, Tenca A, Pohl D, Dvorak K, Goldman A, et al. Barrett's esophagus: proton pump inhibitors and chemoprevention II. Annals of the New York Academy of Sciences 2011 Sep;1232:114-39.

4. El-Serag HB, Sweet S, Winchester CC, Dent J. Update on the epidemiology of gastrooesophageal reflux disease: a systematic review. Gut 2014 Jun;63(6):871-80. 
5. Castell DO, Murray JA, Tutuian R, Orlando RC, Arnold R. Review article: the pathophysiology of gastro-oesophageal reflux disease - oesophageal manifestations. Alimentary pharmacology \& therapeutics 2004 Dec;20 Suppl 9:14-25.

6. Woodland P, Sifrim D. The refluxate: The impact of its magnitude, composition and distribution. Best practice \& research Clinical gastroenterology 2010 Dec;24(6):861-71.

7. Kahrilas PJ, Shaheen NJ, Vaezi MF, American Gastroenterological Association I, Clinical P, Quality Management C. American Gastroenterological Association Institute technical review on the management of gastroesophageal reflux disease. Gastroenterology 2008 Oct;135(4):1392-413, 413 e1-5.

8. Fuchs KH, Babic B, Breithaupt W, Dallemagne B, Fingerhut A, Furnee E, et al. EAES recommendations for the management of gastroesophageal reflux disease. Surgical endoscopy 2014 Jun;28(6):1753-73.

9. Bredenoord AJ, Weusten BL, Curvers WL, Timmer R, Smout AJ. Determinants of perception of heartburn and regurgitation. Gut 2006 Mar;55(3):313-8.

10. Savarino E, Zentilin P, Frazzoni M, Cuoco DL, Pohl D, Dulbecco P, et al. Characteristics of gastro-esophageal reflux episodes in Barrett's esophagus, erosive esophagitis and healthy volunteers. Neurogastroenterology and motility : the official journal of the European Gastrointestinal Motility Society 2010 Oct;22(10):1061-e280.

11. Koek GH, Sifrim D, Lerut T, Janssens J, Tack J. Multivariate analysis of the association of acid and duodeno-gastro-oesophageal reflux exposure with the presence of oesophagitis, the severity of oesophagitis and Barrett's oesophagus. Gut 2008 Aug;57(8):1056-64.

12. Ashton MG, Holdsworth CD, Ryan FP, Moore M. Healing of gastric ulcers after one, two, and three months of ranitidine. Br Med J (Clin Res Ed) 1982 Feb 13;284(6314):467-8.

13. Fineberg HV, Pearlman LA. Surgical treatment of peptic ulcer in the United States. Trends before and after the introduction of cimetidine. Lancet 1981 Jun 13;1(8233):1305-7.

14. Colin-Jones DG. The role and limitations of H2-receptor antagonists in the treatment of gastro-oesophageal reflux disease. Alimentary pharmacology \& therapeutics 1995;9 Suppl 1:9-14.

15. Lachman L, Howden CW. Twenty-four-hour intragastric $\mathrm{pH}$ : tolerance within 5 days of continuous ranitidine administration. The American journal of gastroenterology 2000 Jan;95(1):5761.

16. Furuta K, Adachi K, Komazawa Y, Mihara T, Miki M, Azumi T, et al. Tolerance to H2 receptor antagonist correlates well with the decline in efficacy against gastroesophageal reflux in patients with gastroesophageal reflux disease. Journal of gastroenterology and hepatology 2006 Oct;21(10):1581-5.

17. Sandvik AK, Brenna E, Waldum HL. Review article: the pharmacological inhibition of gastric acid secretion--tolerance and rebound. Alimentary pharmacology \& therapeutics 1997 Dec;11(6): 1013-8.

18. Sachs G. Proton pump inhibitors and acid-related diseases. Pharmacotherapy 1997 JanFeb;17(1):22-37.

19. Savarino V, Di Mario F, Scarpignato C. Proton pump inhibitors in GORD An overview of their pharmacology, efficacy and safety. Pharmacological research 2009 Mar;59(3):135-53.

20. Galmiche JP, Hatlebakk J, Attwood S, Ell C, Fiocca R, Eklund S, et al. Laparoscopic antireflux surgery vs esomeprazole treatment for chronic GERD: the LOTUS randomized clinical trial. JAMA : the journal of the American Medical Association 2011 May 18;305(19):1969-77.

21. Chiba N, De Gara CJ, Wilkinson JM, Hunt RH. Speed of healing and symptom relief in grade II to IV gastroesophageal reflux disease: a meta-analysis. Gastroenterology 1997 Jun;112(6):1798-810.

22. Huang JQ, Hunt RH. Pharmacological and pharmacodynamic essentials of H(2)-receptor antagonists and proton pump inhibitors for the practising physician. Best practice \& research Clinical gastroenterology 2001 Jun;15(3):355-70. 
23. Hershcovici T, Fass R. Management of gastroesophageal reflux disease that does not respond well to proton pump inhibitors. Current opinion in gastroenterology 2010 Jul;26(4):367-78.

24. Fass R, Sifrim D. Management of heartburn not responding to proton pump inhibitors. Gut 2009 Feb;58(2):295-309.

25. Fass R. Proton-pump inhibitor therapy in patients with gastro-oesophageal reflux disease: putative mechanisms of failure. Drugs 2007;67(11):1521-30.

26. Sifrim D, Zerbib F. Diagnosis and management of patients with reflux symptoms refractory to proton pump inhibitors. Gut 2012 Sep;61(9):1340-54.

27. de Bortoli N, Martinucci I, Bellini M, Savarino E, Savarino V, Blandizzi C, et al. Overlap of functional heartburn and gastroesophageal reflux disease with irritable bowel syndrome. World journal of gastroenterology: WJG 2013 Sep 21;19(35):5787-97.

28. Savarino V, Savarino E, Parodi A, Dulbecco P. Functional heartburn and non-erosive reflux disease. Digestive diseases 2007;25(3):172-4.

29. Jenkins H, Sakurai Y, Nishimura A, Okamoto H, Hibberd M, Jenkins R, et al. Randomised clinical trial: safety, tolerability, pharmacokinetics and pharmacodynamics of repeated doses of TAK-438 (vonoprazan), a novel potassium-competitive acid blocker, in healthy male subjects. Alimentary pharmacology \& therapeutics 2015 Apr;41(7):636-48.

- This study evaluates the pharmacological effects of vonoprazan

30. Sachs G, Shin JM, Hunt R. Novel approaches to inhibition of gastric acid secretion. Current gastroenterology reports $2010 \mathrm{Dec} ; 12(6)$ :437-47.

31. van Pinxteren B, Sigterman KE, Bonis P, Lau J, Numans ME. Short-term treatment with proton pump inhibitors, H2-receptor antagonists and prokinetics for gastro-oesophageal reflux disease-like symptoms and endoscopy negative reflux disease. The Cochrane database of systematic reviews 2010(11):CD002095.

32. DeVault KR, Castell DO, American College of G. Updated guidelines for the diagnosis and treatment of gastroesophageal reflux disease. The American journal of gastroenterology 2005 Jan;100(1):190-200.

33. Martinucci I, de Bortoli N, Savarino E, Nacci A, Romeo SO, Bellini M, et al. Optimal treatment of laryngopharyngeal reflux disease. Therapeutic advances in chronic disease 2013 Nov;4(6):287-301.

34. Sachs G, Shin JM, Briving C, Wallmark B, Hersey S. The pharmacology of the gastric acid pump: the $\mathrm{H}+\mathrm{K}+\mathrm{ATPase}$. Annual review of pharmacology and toxicology 1995;35:277-305.

35. Shin JM, Cho YM, Sachs G. Chemistry of covalent inhibition of the gastric (H+, K+)ATPase by proton pump inhibitors. Journal of the American Chemical Society 2004 Jun 30;126(25):7800-11.

36. Robinson $\mathrm{M}$. Review article: the pharmacodynamics and pharmacokinetics of proton pump inhibitors--overview and clinical implications. Alimentary pharmacology \& therapeutics 2004 Nov;20 Suppl 6:1-10.

37. Shin JM, Vagin O, Munson K, Kidd M, Modlin IM, Sachs G. Molecular mechanisms in therapy of acid-related diseases. Cellular and molecular life sciences : CMLS 2008 Jan;65(2):26481.

38. Klotz U, Schwab M, Treiber G. CYP2C19 polymorphism and proton pump inhibitors. Basic \& clinical pharmacology \& toxicology 2004 Jul;95(1):2-8.

39. Furuta T, Shirai N, Sugimoto M, Nakamura A, Hishida A, Ishizaki T. Influence of CYP2C19 pharmacogenetic polymorphism on proton pump inhibitor-based therapies. Drug metabolism and pharmacokinetics 2005 Jun;20(3):153-67.

40. Wedemeyer RS, Blume H. Pharmacokinetic drug interaction profiles of proton pump inhibitors: an update. Drug safety 2014 Apr;37(4):201-11.

41. Bruley des Varannes S, Coron E, Galmiche JP. Short and long-term PPI treatment for GERD. Do we need more-potent anti-secretory drugs? Best practice \& research Clinical gastroenterology $2010 \mathrm{Dec} ; 24(6): 905-21$. 
42. Reimer C. Safety of long-term PPI therapy. Best practice \& research Clinical gastroenterology 2013 Jun;27(3):443-54.

43. Dutta U, Armstrong D. Novel pharmaceutical approaches to reflux disease. Gastroenterology clinics of North America 2013 Mar;42(1):93-117.

44. de Bortoli N, Martinucci I, Savarino E, Bellini M, Bredenoord AJ, Franchi R, et al. Proton pump inhibitor responders who are not confirmed as GERD patients with impedance and $\mathrm{pH}$ monitoring: who are they? Neurogastroenterology and motility : the official journal of the European Gastrointestinal Motility Society 2014 Jan;26(1):28-35.

45. Savarino V, Savarino E, Dulbecco P. Proton pump inhibitor failure: why does it occur and how can it be managed? Digestion 2006;73(4):215-7.

46. de Bortoli N, Martinucci I, Giacchino M, Blandizzi C, Marchi S, Savarino V, et al. The pharmacokinetics of ilaprazole for gastro-esophageal reflux treatment. Expert opinion on drug metabolism \& toxicology 2013 Oct;9(10):1361-9.

47. Munson KB, Sachs $\mathrm{G}$. Inactivation of $\mathrm{H}+, \mathrm{K}+$-ATPase by a $\mathrm{K}+$-competitive photoaffinity inhibitor. Biochemistry 1988 May 31;27(11):3932-8.

48. Keeling DJ, Laing SM, Senn-Bilfinger J. Interactions of SCH 28080 with the gastric (H+ $+\mathrm{K}+$ )-ATPase. Progress in clinical and biological research 1988;273:255-60.

49. Parsons ME, Keeling DJ. Novel approaches to the pharmacological blockade of gastric acid secretion. Expert opinion on investigational drugs 2005 Apr;14(4):411-21.

50. Andersson K, Carlsson E. Potassium-competitive acid blockade: a new therapeutic strategy in acid-related diseases. Pharmacology \& therapeutics 2005 Dec;108(3):294-307.

51. Mendlein J, Sachs G. Interaction of a K(+)-competitive inhibitor, a substituted imidazo[1,2a] pyridine, with the phospho- and dephosphoenzyme forms of $\mathrm{H}+, \mathrm{K}(+)$-ATPase. The Journal of biological chemistry 1990 Mar 25;265(9):5030-6.

52. Wurst W, Hartmann M. Current status of acid pump antagonists (reversible PPIs). The Yale journal of biology and medicine 1996 May-Jun;69(3):233-43.

53. Wallmark B, Briving C, Fryklund J, Munson K, Jackson R, Mendlein J, et al. Inhibition of gastric $\mathrm{H}+, \mathrm{K}+-\mathrm{ATPase}$ and acid secretion by $\mathrm{SCH} 28080$, a substituted pyridyl(1,2a)imidazole. The Journal of biological chemistry 1987 Feb 15;262(5):2077-84.

54. Simon WA, Herrmann M, Klein T, Shin JM, Huber R, Senn-Bilfinger J, et al. Soraprazan: setting new standards in inhibition of gastric acid secretion. The Journal of pharmacology and experimental therapeutics 2007 Jun;321(3):866-74.

55. Ene MD, Khan-Daneshmend T, Roberts CJ. A study of the inhibitory effects of SCH 28080 on gastric secretion in man. British journal of pharmacology 1982 Jul;76(3):389-91.

56. Dent J, Kahrilas PJ, Hatlebakk J, Vakil N, Denison H, Franzen S, et al. A randomized, comparative trial of a potassium-competitive acid blocker (AZD0865) and esomeprazole for the treatment of patients with nonerosive reflux disease. The American journal of gastroenterology 2008 Jan;103(1):20-6.

57. Kim HK, Park SH, Cheung DY, Cho YS, Kim JI, Kim SS, et al. Clinical trial: inhibitory effect of revaprazan on gastric acid secretion in healthy male subjects. Journal of gastroenterology and hepatology 2010 Oct;25(10):1618-25.

58. Shin JM, Inatomi N, Munson K, Strugatsky D, Tokhtaeva E, Vagin O, et al. Characterization of a novel potassium-competitive acid blocker of the gastric H,K-ATPase, 1-[5-(2fluorophenyl)-1-(pyridin-3-ylsulfonyl)-1H-pyrrol-3-yl]-N-methylmethanamin e monofumarate (TAK-438). The Journal of pharmacology and experimental therapeutics 2011 Nov;339(2):412-20.

59. Hori Y, Imanishi A, Matsukawa J, Tsukimi Y, Nishida H, Arikawa Y, et al. 1-[5-(2Fluorophenyl)-1-(pyridin-3-ylsulfonyl)-1H-pyrrol-3-yl]-N-methylmethanamin e monofumarate (TAK-438), a novel and potent potassium-competitive acid blocker for the treatment of acid-related diseases. The Journal of pharmacology and experimental therapeutics 2010 Oct;335(1):231-8. 
60. Keeling DJ, Taylor AG, Schudt C. The binding of a $\mathrm{K}+$ competitive ligand, 2-methyl,8(phenylmethoxy)imidazo(1,2-a)pyridine 3-acetonitrile, to the gastric $(\mathrm{H}++\mathrm{K}+$ )-ATPase. The Journal of biological chemistry 1989 Apr 5;264(10):5545-51.

61. Matsukawa J, Hori Y, Nishida H, Kajino M, Inatomi N. A comparative study on the modes of action of TAK-438, a novel potassium-competitive acid blocker, and lansoprazole in primary cultured rabbit gastric glands. Biochemical pharmacology 2011 May 1;81(9):1145-51.

62. Scott DR, Munson KB, Marcus EA, Lambrecht NW, Sachs G. The binding selectivity of vonoprazan (TAK-438) to the gastric $\mathrm{H}+, \mathrm{K}+$-ATPase. Alimentary pharmacology \& therapeutics 2015 Dec;42(11-12):1315-26.

63. Hori Y, Matsukawa J, Takeuchi T, Nishida H, Kajino M, Inatomi N. A study comparing the antisecretory effect of TAK-438, a novel potassium-competitive acid blocker, with lansoprazole in animals. The Journal of pharmacology and experimental therapeutics 2011 Jun;337(3):797-804.

64. Arikawa Y, Nishida H, Kurasawa O, Hasuoka A, Hirase K, Inatomi N, et al. Discovery of a novel pyrrole derivative 1-[5-(2-fluorophenyl)-1-(pyridin-3-ylsulfonyl)-1H-pyrrol-3-yl]-Nmethylmethanamin e fumarate (TAK-438) as a potassium-competitive acid blocker (P-CAB). Journal of medicinal chemistry 2012 May 10;55(9):4446-56.

65. Sakurai Y, Nishimura A, Kennedy G, Hibberd M, Jenkins R, Okamoto H, et al. Safety, Tolerability, Pharmacokinetics, and Pharmacodynamics of Single Rising TAK-438 (Vonoprazan) Doses in Healthy Male Japanese/non-Japanese Subjects. Clinical and translational gastroenterology 2015;6:e94.

66. Sakurai Y, Mori Y, Okamoto H, Nishimura A, Komura E, Araki T, et al. Acid-inhibitory effects of vonoprazan $20 \mathrm{mg}$ compared with esomeprazole $20 \mathrm{mg}$ or rabeprazole $10 \mathrm{mg}$ in healthy adult male subjects--a randomised open-label cross-over study. Alimentary pharmacology \& therapeutics 2015 Sep;42(6):719-30.

- This randomized, comparative, cross-over study demonstrates that vonoprazan exerts a more rapid and sustained acid-inhibitory effect compared to the second generation of PPIs (esomeprazole or rabeprazole)

67. Kagami T, Sahara S, Ichikawa H, Uotani T, Yamade M, Sugimoto M, et al. Potent acid inhibition by vonoprazan in comparison with esomeprazole, with reference to CYP2C19 genotype. Alimentary pharmacology \& therapeutics 2016 May;43(10):1048-59.

- Differently from Esomeprazole, this study shows that the acid-inhibitoy effect of vonoprazan is not significantly influenced by the CYP2C19 genetic polymorphisms

68. Ashida K, Sakurai Y, Hori T, Kudou K, Nishimura A, Hiramatsu N, et al. Randomised clinical trial: vonoprazan, a novel potassium-competitive acid blocker, vs. lansoprazole for the healing of erosive oesophagitis. Alimentary pharmacology \& therapeutics 2016 Jan;43(2):240-51.

- This is the first study comparing vonoprazan vs lansoprazole in erosive esophagitis patients and demonstrating that vonoprazan was effective and non-inferior to lansoprazole in healing esophagitis at low and high doses.

69. Ashida K, Sakurai Y, Nishimura A, Kudou K, Hiramatsu N, Umegaki E, et al. Randomised clinical trial: a dose-ranging study of vonoprazan, a novel potassium-competitive acid blocker, vs. lansoprazole for the treatment of erosive oesophagitis. Alimentary pharmacology \& therapeutics 2015 Sep;42(6):685-95.

- This study comparing vonoprazan vs lansoprazole in erosive esophagitis patients revealed that the proportion of patients with healed erosive esophagitis tended to be higher in the vonoprazan group, as compared with lansoprazole, in patients with more severe esophagitis (grades C/D) as well as in those classified as CYP2C19 extensive metabolisers. 